\title{
Gut origin of sepsis: a prospective study investigating associations between bacterial translocation, gastric microflora, and septic morbidity
}

\author{
J MacFie, C O’Boyle, C J Mitchell, P M Buckley, D Johnstone, P Sudworth
}

\begin{abstract}
Aims-To investigate the "gut origin of sepsis" hypothesis.

Methods-Prospective controlled study of 279 surgical patients in which cultures of nasogastric aspirates were compared with those obtained from mesenteric lymph nodes taken at laparotomy and the organisms cultured from subsequent septic complications. Bacterial translocation was confirmed if positive cultures were obtained from mesenteric lymph nodes. Postoperative sepsis was defined as any positive culture in the postoperative period. Bacterial species obtained in gastric microflora, mesenteric lymph nodes, and postoperative septic complications were compared. Results-Only $85 / 279$ patients $(31 \%)$ had a sterile nasogastric aspirate; the most frequently identified organism was Candida spp. (54\%) and the most common enteric organism cultured was $E$ coli $(20 \%)$. Multiple organisms were isolated in $39 \%$ and occurred more frequently in patients aged over 70 years, those undergoing nonelective surgery, and in those requiring proximal gastrointestinal surgery. Postoperative sepsis was more common in these patients. Bacterial translocation occurred in $21 \%$ and was significantly more frequent in those with multiple organisms in their nasogastric aspirates. $E$ coli was the commonest organism isolated from the lymph node specimens (48\%) and septic foci $(53 \%)$. Fungal translocation did not occur. An identical genus was identified in the nasogastric aspirate and the septic focus in $30 \%$ of patients, in the nasogastric aspirate and the lymph node in $31 \%$, and in the lymph node and a postoperative septic focus in $45 \%$.

Conclusions-Proximal gut colonisation is associated with both increased bacterial translocation and septic morbidity. The commonality of organisms identified supports the gut origin of sepsis hypothesis. (Gut 1999;45:223-228)
\end{abstract}

Keywords: gut origin of sepsis; bacterial translocation; septic morbidity

The "gut origin of sepsis" hypothesis proposes that bacteria, which are normally resident within the lumen of the intestinal tract, translocate across the intestinal epithelial barrier and act as a source of sepsis at distant sites. ${ }^{12}$ Many animal studies support this concept. $^{34}$ A number of factors have been shown to predispose to bacterial translocation. These include shock with reduced splanchnic blood flow, parenteral nutrition, intestinal epithelial damage, and antibiotic therapy. ${ }^{4}$

The clinical significance of bacterial translocation remains unclear although there is increasing circumstantial evidence to suggest that it may play an important role in the causation of sepsis. For example, it is well recognised that nosocomial infection is frequently a consequence of gut derived organisms, such as Escherichia coli. ${ }^{5}$ Furthermore, many authors report an increased incidence of septic complications in patients receiving parenteral as opposed to enteral nutrition and this, it is suggested, could be accounted for by alterations in intestinal barrier function predisposing to bacterial translocation. ${ }^{67}$ Finally, an association between upper gastrointestinal microflora and subsequent septic complications has been explained on the basis of bacterial translocation. ${ }^{8}$ In critically ill patients, colonisation of the upper gastrointestinal tract with both enteric and nonenteric organisms may predispose to nosocomial infection. ${ }^{9}$

Despite this large amount of evidence implicating intestinally derived bacteria as the cause of septic morbidity, there are no published clinical studies which confirm that this occurs as a consequence of bacterial translocation. Our aim was to investigate changes in upper gastrointestinal microflora and to determine whether there were any associations between such changes and the occurrence of bacteriologically confirmed bacterial translocation and subsequent septic morbidity.

\section{Patients and methods}

UPPER GASTROINTESTINAL MICROFLORA

Nasogastric aspirates were obtained from 279 surgical patients for microbiological analysis. Table 1 summarises clinical diagnoses and surgical procedures. The median age of the group was 66 years (range 8-94 years) with a slight preponderance of male subjects (150:129). The majority of these patients were about to undergo laparotomy (87\%) and 152 patients

Abbreviations used in this paper: BT, bacterial translocation; CLED, cysteine lactose electrolyte deficient; ICU, intensive care unit; MACS, modular atmosphere controlled system; MLN, mesenteric lymph node. 
Table 1 Details of patients

\begin{tabular}{lrlr}
\hline Diagnoses $^{*}$ & $n$ & Surgical procedures** & $n$ \\
\hline Upper gastrointestinal & & Gastrectomy & 4 \\
$\quad$ cancer & 30 & & \\
Peptic ulcer & 12 & & \\
Cholelithiasis & 18 & Cholecystectomy & 4 \\
Acute pancreatitis & 30 & & \\
Pancreatic cancer & 10 & Pancreatectomy & 4 \\
Crohn's disease & 38 & Small bowel resection & 18 \\
Ulcerative colitis & 26 & Colectomy/pouch & 18 \\
Cancer of & & Colectomy & 75 \\
$\quad$ colon/rectum & 75 & & \\
Diverticular disease & 10 & & \\
Appendicitis & 5 & & \\
Aortic aneurysm & 14 & Aneurysm repair & 2 \\
Miscellaneous & 11 & Miscellaneous & 136 \\
Total & 279 & Total & \\
\hline
\end{tabular}

*All patients who had nasogastric aspirates taken.

${ }^{\star \star}$ All patients in whom mesenteric lymph nodes were sampled.

were acute admissions. Patients were excluded if they had received antibiotics, proton pump inhibitors, $\mathrm{H}_{2}$ receptor antagonists, or enteral or parenteral nutrition in the month prior to admission. All patients were fasted for a minimum of eight hours prior to surgery. Aspirates were taken after induction of general anaesthesia, before commencement of surgery. In the 36 patients who did not undergo surgery, aspirates were obtained immediately following insertion of a nasogastric tube which was deemed necessary as part of their conservative management: the majority of these patients were admitted with acute pancreatitis, cholelithiasis, or exacerbations of inflammatory bowel disease. None of these patients were receiving enteral nutrition at the time of sampling.

In all patients, $15 \mathrm{ml}$ of nasogastric aspirate was aspirated into a sterile syringe immediately after passing a sterile nasogastric Ryle's tube. The first $5 \mathrm{ml}$ were discarded and the remaining $10 \mathrm{ml}$ were immediately sent for culture and sensitivity analysis. This method of assessing gastric microflora has been previously shown to produce reliable and reproducible results. ${ }^{10}$

\section{BACTERIAL TRANSLOCATION}

In 136 patients (median age 66 years, range 19-94; 65 men, 71 women), at the start of laparotomy a serosal scraping was obtained from the antimesenteric border of the terminal ileum. A lymph node from the mesentery of the terminal ileum was excised by the use of a separate clean surgical blade. The samples were transported promptly in separate containers in sterile saline solution for microbiological analysis. Simultaneously, a peripheral blood sample was obtained by means of venepuncture. This was placed into aerobic and anaerobic blood culture bottles. Prophylactic antibiotics were only given to the patients after these samples were obtained.

Samples of serosa or mesenteric lymph nodes were not taken from patients in whom there was evidence of peritoneal contamination. This method of harvesting mesenteric lymph nodes and serosa for microbiological assessment of bacterial translocation has been extensively used in previous studies. ${ }^{11} 12$
SEPTIC MORBIDITY

The postoperative course of all patients was closely monitored until the time of discharge or death. Cultures of blood, sputum, or urine were taken whenever deemed clinically appropriate. All wound discharges of whatever nature were cultured as were any fluid collections identified on imaging in the postoperative course.

\section{MICROBIOLOGICAL TECHNIQUES}

Both serosal scrapings and the lymph nodes were separately homogenised in saline by means of a stomacher (Seward Medical, London, UK). Each homogenate was inoculated onto Columbia blood agar and cysteine lactose electrolyte deficient (CLED) media for aerobic incubation and incubated in air $+5 \%$ carbon dioxide and onto Wilkins-Chalgren blood agar with neomycin and Columbia blood agar for anaerobic culture. Anaerobic cultures were incubated in a Don Whitley modular atmosphere controlled system (MACS) cabinet in an atmosphere of $80 \%$ nitrogen, $10 \%$ hydrogen, and $10 \%$ carbon dioxide. Aerobic cultures were incubated at $37^{\circ} \mathrm{C}$ for two days and anaerobic cultures were incubated at $37^{\circ} \mathrm{C}$ for five days.

Blood cultures-Aerobic and anaerobic blood culture bottles were incubated constantly at $37^{\circ} \mathrm{C}$ for five days. BACTEC culture vial types Plus Aerobic/F and Plus Anaerobic/F were each inoculated with $10 \mathrm{ml}$ of blood. Each vial contains a chemical sensor which can detect increases in carbon dioxide produced by the growth of microorganisms. Bacterial growth in the blood culture bottles was detected on a Bactec 9240 instrument (Becton Dickinson Europe, Meylan Cedex, France). All blood culture vials with positive readings had $0.5 \mathrm{ml}$ of media extracted which was examined by Gram staining and further inoculated onto Columbia blood agar, chocolatised Columbia blood agar, and CLED media, and incubated in air $+5 \%$ carbon dioxide, and onto Columbia blood agar incubated for 48 hours in an anaerobic atmosphere as previously described. ${ }^{12}$

Nasogastric aspirates, pus, or any identified fluid collections were cultured on Columbia blood agar and CLED media for aerobic incubation and incubated in air $+5 \%$ carbon dioxide and onto Columbia blood agar and Wilkins-Chalgren blood agar with neomycin for anaerobic culture.

Isolates grown from mesenteric lymph nodes, serosal scrapings, and peripheral blood samples were identified by characteristic colonial and microscopic appearances and by using standard microbiological tests. ${ }^{13}{ }^{14}$ Whenever required, full specification of organisms belonging to the family Enterobacteriacae was performed using API 20E strips (bioMerieux France). Enterococcus faecalis and other streptococci were identified by microscopic and colonial appearances, Lancefield grouping by the Streptex kit (Murex UK), and where appropriate by using API rapid ID 32 Strep strips (bioMerieux France). Obligate anaerobic isolates were identified by characteristic colonial 
Table 2 Details of positive cultures obtained from three sites sampled

\begin{tabular}{lrcc}
\hline & $\begin{array}{c}\text { Nasogastric } \\
\text { aspirate } \\
(n=194)\end{array}$ & $\begin{array}{l}\text { Septic focus } \\
(n=89)\end{array}$ & $\begin{array}{c}\text { Lymph node } \\
(n=29)\end{array}$ \\
\hline Candida & $104(54 \%)$ & $10(11 \%)$ & 0 \\
E coli & $38(20 \%)$ & $29(33 \%)$ & $14(48 \%)$ \\
Streptococcus & $33(17 \%)$ & $4(4 \%)$ & \\
Lactobacillus & $27(14 \%)$ & 0 & \\
E faecalis & $23(12 \%)$ & $18(20 \%)$ & $2(10 \%)$ \\
S epidermidis & $17(9 \%)$ & $11(12 \%)$ & $11(20 \%)$ \\
Enterobacter & $17(9 \%)$ & 0 & $1(7 \%)$ \\
Klebsiella & $13(7 \%)$ & $4(4 \%)$ & 1 \\
Citrobacter & $12(6 \%)$ & $1(1 \%)$ & \\
Diptheroids & $12(6 \%)$ & $1(1 \%)$ & 1 \\
Staph aureus & $10(5 \%)$ & 0 & \\
Neisseria & $10(5 \%)$ & 0 & \\
Pseudomonas & $9(5 \%)$ & $15(17 \%)$ & \\
Proteus & $9(5 \%)$ & $8(9 \%)$ & $2(10 \%)$ \\
Bacteroides & $6(3 \%)$ & $6(7 \%)$ & $1(7 \%)$ \\
Bacillus & $6(3 \%)$ & & \\
H influenzae & $6(3 \%)$ & $4(4 \%)$ & \\
Serratia & $4(2 \%)$ & & \\
Morganella & $4(2 \%)$ & & \\
C perfringens & $5(3 \%)$ & & \\
Acinetobacter & $3(2 \%)$ & & \\
Hafnia & $2(1 \%)$ & & \\
\hline & & & \\
\hline
\end{tabular}

growth characteristics, sensitivity to metronidazole, microscopic appearance, and rapid ID32A strips (bioMerieux France). Obligate aerobic isolates and non-fermenting Gram negative bacilli were identified using API 20NE strips (bioMerieux France). Yeasts were identified by characteristic colonial and microscopic appearances and the Auxacolor colorimetric identification system (Sanofi Diagnostics Pasteur, France). Bacillus spp. and coryneforms were identified by standard microbiological tests. Microscopic and macroscopic appearances, catalase reaction, coagulase, and DNAse reaction identified organisms belonging to the genus Staphylococcus.

Postoperative sepsis was defined as the presence of clinical, haematological, or radiographic evidence of infection where positive bacterial culture was obtained from a septic focus.

STATISTICAL ANALYSIS

All data were expressed as the median and interquartile range. Comparisons between non-parametric groups of data were made using the $\chi^{2}$ test or the Mann-Whitney $U$ test.

\section{Results}

GASTRIC MICROFLORA

Nasogastric aspirates were obtained from 279 patients of whom $85(31 \%)$ had a sterile nasogastric aspirate. A single species was identified in $84(30 \%)$ and multiple organisms in $110(39 \%)$. Both enteric and non-enteric pathogens were isolated (table 2). These were usually organisms of low intrinsic virulence which are commonly associated with opportunistic nosocomial infections. The most frequently identified organism was Candida spp. $(54 \%)$, which is not normally found in the healthy gut. The most common enteric organisms to be cultured were E coli (20\%), Lactobacillus spp. (14\%), Enterococcus spp. (12\%), and Enterobacter spp. (9\%). The genus Enterobacteriaecae (enteric Gram negative facultatively anaerobic rods) which includes $E$ coli and
Enterobacter species was present in $35 \%$ of positive cultures.

Multiple organisms were isolated in 39\% of patients. These occurred more commonly in patients aged over 70 years $(50 \%$ versus $32 \%$, $\mathrm{p}<0.01$ ), patients undergoing non-elective surgery $(51 \%$ versus $28 \%, \mathrm{p}<0.001)$, and in those requiring proximal gastrointestinal surgery $(51 \%$ versus $34 \%, \mathrm{p}<0.01)$. The presence of distal bowel obstruction was associated with increased colonisation but this was not significant $(59 \%$ versus $37 \%, p<0.08)$. There was no association between upper gastrointestinal colonisation and malignancy, weight loss prior to surgery, or the administration of preoperative parenteral or enteral nutrition.

\section{BACTERIAL TRANSLOCATION}

In 136 of the patients who had nasogastric aspirates taken prior to laparotomy a mesenteric lymph node was sampled for assessment of translocation. Bacterial translocation occurred in 29 patients (21\%) and was significantly more frequent in those who were found to have multiple organisms in their nasogastric aspirates $(\mathrm{p}<0.01)$.

A total of 33 organisms were cultured from 29 positive nodal samples (see table 2) and multiple organisms were cultured in five of these patients. Fungal translocation did not occur in this series. $E$ coli was the most commonly identified organism and was found in $14(48 \%)$ patients. The remainder consisted of organisms frequently associated with nosocomial infection.

Only one patient in this series had a positive blood culture ( $E$ coli) at the time of sampling.

\section{POSTOPERATIVE SEPSIS}

The incidence of positive bacterial cultures in the postoperative period was high in this series, occurring in $89 / 279$ patients (32\%). Thirty six of these 89 patients $(40 \%)$ developed urinary sepsis, $31(35 \%)$ respiratory infection, and 27 $(30 \%)$ wound sepsis. Seven patients $(8 \%)$ had postoperative positive blood cultures and four (4\%) developed an infected intra-abdominal collection.

Table 2 summarises the spectrum of bacteria cultured from postoperative septic foci. Coliform (enteric) organisms were responsible for sepsis in 58/89 patients (65\%). E coli was the commonest organism $(\mathrm{p}<0.001)$ isolated from septic foci and was present in $33 \%$ of cases.

ASSOCIATIONS BETWEEN GASTRIC MICROFLORA, BACTERIAL TRANSLOCATION, AND SEPTIC MORBIDITY

Translocation - gastric microflora

Bacterial translocation occurred in 29 of the 136 patients sampled. In 86 of these, nasogastric aspirates were sterile or yielded a single organism only. The translocation prevalence in this group was $14 \%$ (12/86 patients). The remaining 50 patients in this group who had MLN sampling grew multiple organisms from their gastric aspirates. In these the translocation prevalence was $34 \%$ (17/50 patients). This difference is statistically significant $(\mathrm{p}<0.02)$. 
Table 3 Translocation and septic morbidity

\begin{tabular}{|c|c|c|c|}
\hline Lymph node & Septic focus & Clinical sepsis & Nasogastric aspirate \\
\hline E coli ${ }^{\star \star}$ & E coli/C albicans & UTI/RTI & E coli $/ C$ albicans $/ P$ vulgaris \\
\hline$E \operatorname{coli}^{\star}$ & E coli & UTI & E cloacae/Aeromonas \\
\hline$E \operatorname{coli}^{\star \star}$ & E coli/S faecalis & Wound & E coli / K oxytoca/C albicans \\
\hline S epidermidis $\star$ & $S$ epidermidis & Septicaemia & E cloacae/Serratia/Morganella \\
\hline P mirabilis $\star \star$ & $P$ mirabilis/S aureus & UTI/RTI & $P$ mirabilis $/ S$ aureus $/ C$ albicans \\
\hline Koxytoca & E coli & UTI & Diphtheroids/S aureus \\
\hline Bacillus & E coli & Wound & Bacillus/C albicans \\
\hline S epidermidis & $B$ fragilis & Wound & Nil \\
\hline$S$ epidermidis & E coli & UTI & E cloacae \\
\hline Sepidermidis & E coli/P aeruginosa & UTI/septicaemia & $E$ coli/Lactobacillus/ $C$ freundii \\
\hline$S$ epidermidis & E coli & Wound & H influenzae \\
\hline
\end{tabular}

Eleven patients who exhibited evidence of translocation developed septic complications. ${ }^{\star}$ Five of these grew the same organism type from both the nodal and septic specimens. ${ }^{\star \star}$ In three patients the same organism was also isolated from their nasogastric aspirates. UTI, urinary tract infection; RTI, respiratory tract infection.

The same bacterial genus was found in the nasogastric aspirate and the lymph node in nine of these 29 patients (31\%). The organisms identified were E coli (4), Proteus spp. (2), Bacillus spp. (1), and coagulase negative staphylococci (2).

\section{Translocation-septic foci}

Postoperative sepsis was more common in translocators than non-translocators (11/29 $(38 \%)$ versus $32 / 107$ (30\%), NS). Of those patients with evidence of translocation to lymph nodes and who subsequently developed postoperative sepsis $(n=11)$, five $(45 \%)$ grew the same genus of bacteria from both the peroperative nodal sample and the postoperative septic focus. $E$ coli was the commonest organism to be isolated from lymph node specimens (48\%) and septic foci (33\%).

\section{Gastric microflora-septic foci}

Eighty five patients $(30.5 \%)$ had a sterile nasogastric aspirate and $13(15.3 \%)$ of these developed postoperative sepsis. A single organism was cultured in 84 patients $(30.1 \%)$ and sepsis developed in 21 (25\%) of these. Multiple organisms were identified in 110 patients $(39.4 \%)$ and $55(50 \%)$ of these had postoperative sepsis. The difference in the incidence of postoperative sepsis between those with sterile cultures and those with multiple organisms is statistically significant $(\mathrm{p}<0.01)$. Patients developing urinary, respiratory, or wound sepsis grew multiple organisms from nasogastric aspirates in $56 \%, 65 \%$, and $59 \%$ respectively compared with $29 \%$ for patients who did not develop septic complications $(\mathrm{p}<0.01)$. Candida spp. (54\%) was the commonest organism identified from nasogastric aspirates and was the predominant organism in $10 \%$ of postoperative septic complications. Eighty nine of the 279 patients $(32 \%)$ developed sepsis. In 27 $(30 \%)$ of these an identical genus was identified in the nasogastric aspirate and the septic focus.

In three of the 11 patients with bacterial translocation who also developed postoperative sepsis the same bacterial genus was cultured from all three sites (lymph node, nasogastric aspirate, and septic focus). The responsible organisms were E coli (2) and Proteus mirabilis (1). Table 3 summarises these results.

\section{Discussion}

The results of this study indicate that the upper gastrointestinal tract is sterile in a minority of surgical patients. In this series bacterial colonisation occurred most frequently in elderly patients with gastric or small intestinal pathology requiring urgent surgery. Gastric colonisation, and in particular the occurrence of multiple organisms, was associated with a significant increase in septic complications which were of predominantly enteric bacterial origin. Our results also show that gastric colonisation itself predisposes to translocation. Although association does not prove causation, the frequency with which organisms of identical genus were identified in nasogastric aspirates, mesenteric lymph nodes, and subsequently in the postoperative septic focus supports the gut origin of sepsis hypothesis. Figure 1 illustrates the commonality of organisms.

The human gastrointestinal tract contains a wide variety of aerobic and anaerobic bacteria. Unique flora reside in each section of the intestine and are prevented from migrating to extraintestinal sites by a single epithelial layer on the mucosa. The upper gut and stomach are usually sterile or sparsely populated with relatively avirulent bacteria. ${ }^{10}$ These are most commonly Gram positive and aerobic and the most frequently isolated species are streptococci, staphylococci, lactobacilli, and various fungi. ${ }^{15}$ In the distal ileum Gram negative bacteria outnumber Gram positive organisms. Enterobacteriaceae predominate and anaerobic bacteria are found in substantial numbers. ${ }^{16} 17$

This study confirms that critical illness is often associated with significant proximal gut overgrowth of enteric organisms which may contribute to nosocomial infection. ${ }^{89}{ }^{18}$ The similarity in the spectrum of organisms identified in septic foci and those cultured from gastric aspirates is suggestive that the infecting

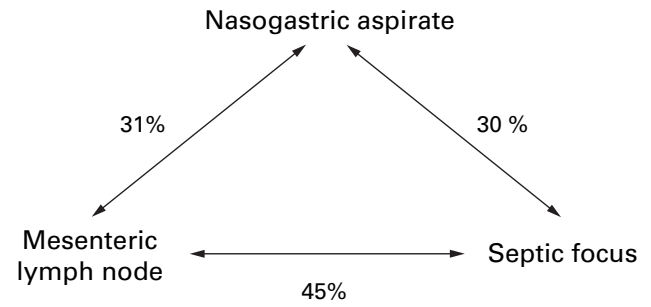

Figure 1 Commonality of organisms from three culture sites. 
organisms are of gut origin. Marshall et al showed that over $90 \%$ of their ICU patients with infection had at least one episode of infection with an organism that was simultaneously present in the upper gastrointestinal tract. ${ }^{8}$ In their series colonisation with Candida, Pseudomonas, Staphylococcus epidermidis, and Streptococcus faecalis were the commonest organisms responsible. A study in neonates defined microbial translocation as having occurred if organisms isolated from blood samples were also carried in the throat or rectum. ${ }^{18}$ They reported six infants with 15 episodes of septicaemia attributable to enteric organisms. In our study $E$ coli, Klebsiella, Candida, and enterococci were most frequently implicated. Our results confirm the preponderance of colonisation by Candida and $E$ coli and also show a similar spectrum of organisms colonising the upper gut as were later identified in septic foci. In addition, our study also shows that the magnitude of colonisation is important. Colonisation with multiple organisms was associated with a significant increase not only in septic complications but also with bacterial translocation. The prognostic value of nasogastric aspirate culture in the management of the critically ill patient merits further investigation.

It is a remarkable testimony to the efficiency of the intestinal barrier that large populations of indigenous bacteria reside in the healthy lower gut without causing harm. This indigenous microflora exerts an important influence in preventing colonisation with exogenous pathogens, so called colonisation resistance. ${ }^{19}$ Many factors have been implicated in the regulation of the different populations of microflora. These include the creation of "microclimates" whereby facultative bacteria, for example, utilise oxygen, thereby ensuring a suitable environment for obligate anaerobes. ${ }^{16}$ This has prompted suggestions that obligate anaerobic bacteria may be the principle inhibitors of translocation of $E$ coli and other potentially pathogenic bacteria. ${ }^{20}{ }^{21}$ Disruption of the normal flora may predispose to a breakdown in colonisation resistance. This may occur, for example, with antibiotic therapy and there is evidence that substrate utilisation by different organisms will also influence other bacterial concentrations. ${ }^{22} 23$ These factors cannot be implicated in this study as patients were excluded if antibiotics or artificial nutrition had been used in the month prior to admission. It would seem, therefore, that pathological colonisation of the upper gut can occur simply as a consequence of gastrointestinal pathology or serious illness. Bacterial translocation was associated with an increased incidence of septic complications. However, although this difference was not significant we have previously shown that bacterial translocation is associated with a significant increase in septic morbidity in similar patients to those reported here. ${ }^{12}$ The methodology used to establish bacterial translocation almost certainly underestimates the true incidence of this phenomenon. Practical and ethical constraints preclude the sampling of multiple nodes. Despite these logistical difficulties culture of mesenteric lymph nodes pro- vides firm evidence of translocation, whereas the converse is not true. In this series, Gram negative facultative anaerobes, and particularly $E$ coli, were the most common organisms identified in mesenteric lymph nodes. Obligate anaerobes were not identified in this series, but are known to translocate, so absolute intraluminal population density would therefore not seem to have a significant influence on bacterial translocation. ${ }^{12}$ Previous authors have also reported a low incidence of bacterial translocation by obligate anaerobes. ${ }^{19} 21$ This finding would also suggest that translocation rates cannot be explained by alterations in gut permeability alone. ${ }^{24}$

This study confirms that critical illness is associated with proximal gut overgrowth and that this predisposes to septic morbidity. A purely descriptive study such as this cannot establish causation with certainty. It could be argued that gut colonisation is a consequence of infection and a manifestation of microbial dissemination from the initial focus of infection. Indwelling urinary or venous catheters are known to be associated with septic morbidity and the presence of a nasogastric tube may itself predispose to infection by permitting aspiration. In this study sampling of gastric aspirate and mesenteric lymph nodes occurred either on admission or at the time of surgery before indwelling catheters had been present for any prolonged period. Further, in this series, there was a significant increase in the incidence of multiorganism colonisation in elderly patients with acute conditions. These patients must have been immunosuppressed to some extent and it is possible that this is the common factor that predisposes the patient to both gastric colonisation as well as subsequent sepsis.

While accepting these caveats the evidence from this study does lend support to the gut origin of sepsis theory. Firstly, it is improbable that organisms cultured from mesenteric lymph nodes originated from anywhere other than the gut lumen as patients with peritoneal contamination were excluded. Ethical constraints precluded routine sampling of portal venous blood but systemic cultures were routinely obtained and only one was positive. In the absence of persistent bacteraemia we consider it probable that organisms cultured from mesenteric nodes must have arisen from the gut lumen. Secondly, the presence of bacterial translocation was associated with an increase in septic complications (38\% versus $30 \%)$. Previous studies have confirmed a significant correlation between bacterial translocation and septic morbidity. ${ }^{12}$ Thirdly, the commonality of organisms identified from nasogastric aspirates, mesenteric lymph nodes, and septic foci supports the hypothesis. The organisms responsible for postoperative infections in this series were all gut pathogens which have been shown to translocate and furthermore, the commonest, the Enterobacteriaceae, were also responsible for postoperative septic complications in 63\% (27/43) of cases. Of those patients in whom bacteria were cultured from mesenteric lymph nodes and who also subsequently developed postoperative sep- 
sis, $45 \%(5 / 11)$ grew at least one similar species type at both sites.

Enteric bacteria and particularly the Enterobacteriacae are the most frequently implicated bacteria in the gut origin of sepsis hypothesis and these organisms predominated in our study at all three sites sampled. These associations may have occurred simply because Enterobacteriacae are commonly found in the gut and are frequent pathogens. This, in our view, is unlikely; the commonality of organisms shown between the three separate culture sites is not what would be anticipated if concentrations within the gut were the major factor in the development of subsequent sepsis. Further, no evidence for candidal translocation was found in this study despite its prevalence in both nasogastric aspirates (54\%) and postoperative septic foci $(11 \%)$. Others have reported that Candida in sufficient numbers can migrate across a normal intestinal mucosal barrier and that translocation occurs after bolus ingestion. ${ }^{25}{ }^{26}$ The absence of candidal translocation in our series suggests that luminal concentration alone is unlikely to be the only predisposing factor to translocation. Virulence factors and local conditions within the lumen of the intestine may well play an as yet undefined role in the processs of translocation. It must also be recognised that translocation is unlikely to account for all septic morbidity. Our failure to show Candida in mesenteric lymph nodes suggests that candidal infestation may well occur by means other than translocation. It is well established, for example, that aspiration of gastric Candida may explain fungal pneumonias.

In conclusion, we recognise that the associations shown in this descriptive study do not prove causation with respect to the gut origin of sepsis hypothesis. However, absolute proof that intestinally derived bacteria translocate and result in sepsis would necessitate precise phage typing of all gastrointestinal flora on a daily basis with concomitant culture of all subsequent septic foci to show commonality and a temporal relation. The practical and ethical difficulties of establishing such a clinical study are obvious. This study supports the gut origin of sepsis hypothesis and suggests a pathological significance to the phenomenon of bacterial translocation. Taken with other reports we consider that bacterial translocation probably occurs in all individuals but is not clinically significant in the presence of a fully functional immune system. Destruction of translocated bacteria in mesenteric lymph nodes is dependent on the immunological competence of the host and individual bacterial virulence factors. In severely immunocompromised individuals, viable translocated bacteria are propagated to extraintestinal sites as potential foci of sepsis. Bacterial translocation seems to be an important early step in the promotion of sepsis in debilitated postoperative patients. Elucidation of the defence mechanisms by which intestinal microflora are confined within the gut lumen will be a significant advance in the understanding of gut derived infection.

1 Saadia R, Schein M, MacFarlane C, et al. Gut barrier function and the surgeon. Br F Surg 1990;77:487-92.

2 Deitch EA. Multiple organ failure. Ann Surg 1992;216:11734.

3 Ahrendt GT, Barbul A. Nutrition and multiple organ dysfunction syndrome. Curr Opin Gastroenterol 1994;10: 203-9.

4 Deitch EA. Nutrition and the gut mucosal barrier. Curr Opin Gen Surg 1993:85-91.

5 Emori TG, Gaynes RP. An overview of nosocomial infections, including the role of the microbiology laboratory. Clin Microbiol Rev 1993;6:428-44.

6 Moore FA, Moore EE. Early post injury enteral nutrition: Moore FA, Moore EE. Early post injury enteral nutrition:
does it make a difference? In: Vincent JL, editor. Yearbook of intensive care and emergency medicine. New York: Springer Verlag, 1996:250-9.

7 Kudsk KA, Croce MA, Fabian TC, et al. Enteral versus parenteral feeding. Ann Surg 1992;215:503-13.

8 Marshall JC, Christou NV, Meakins JL. The gastrointestinal tract; the undrained abscess of multiple organ failure. Ann Surg 1993;218:111-19.

9 Marshall JC, Christou NV, Horn H, et al. The microbiology of multiple organ failure: the proximal GI tract as an occult reservoir of pathogens. Arch Surg 1988;123:309-15.

10 Gorbach SL, Plaut AG, Nahas L, et al. Studies of intestinal microflora. II. Micro-organisms of the small intestine and their relations to oral and faecal flora. Gastroenterology 1967;53:856-67.

11 Sedman PC, MacFie J, Sagar P, et al. The prevalence of gut translocation in humans. Gastroenterology 1994;107:643-9.

2 O'Boyle C, MacFie J, Mitchell CJ, et al. The microbiology of bacterial translocation in humans. Gut 1998;42:29-35.

13 Stokes J, Ridgeway GL. Clinical bacteriology. 5th edn. SevenStokes J, Ridgeway GL. Clinical bacter
oaks, UK: Edward Arnold, 1980.

14 Barrow GI, Feltham RK (eds). Cowan and Steel's manual for identification of medical bacteria. 3rd edn. Cambridge: Cambridge University Press, 1993.

15 Drasar BS, Shiner M, McCleod GM. Studies on the intestinal flora. The bacterial flora of the gastrointestinal tract in healthy and achlorhydric persons. Gastroenterology 1969;56: $71-9$.

16 Simon GL, Gorbach SL. The human intestinal microflora. Dig Dis Sci 1986;31:147S-62S.

17 Simon GL, Gorbach SL. Intestinal flora in health and disease. Gastroenterology 1984;86:174-93.

18 Pierro A, Hendrick KF, Donnell SC, et al. Microbial translocation in neonates and infants receiving long-term parenteral nutrition. Arch Surg 1996;131:176-9.

19 Van der Waaij DJM, Berghuis-de Vries JM, Lekkerkerk-van der Wees JEC. Colonization resistance of the digestive tract and the spread of bacteria to the lymphatic organs in mice. f Hygiene 1971;70:335-42.

20 Hentges DJ. Role of the intestinal microflora in the defence against infection. In: Hentges DJ, editor. Human intestinal microflora in health and disease. New York: Academic Press, 1983:311-31.

21 Freter R, Abrams GD. Function of various intestinal bacteria in converting germ-free mice to the normal state. Infect Immun 1972;6:119-26.

22 Mackowiak PA. The normal microbial flora. $N$ Engl f Med 1982;307:83-93.

23 Freter R. In vivo and in vitro antagonism of intestinal bacteria against Shigella flexneri II. The inhibitory mechanism. $\mathcal{f}$ Infect Dis 1962;110:38-46.

24 O'Boyle C, MacFie J, Dave K, et al. Alterations in intestinal barrier function do not predispose to translocation of enteric bacteria in gastroenterological patients. Nutrition 1998;14:358-62.

25 Krause W, Matheis H, Wulf K. Fungaemia and funguria after oral administration of Candida albicans. Lancet

26 Stone HH, Kolb LD, Currie CA, et al. Candida sepsis: pathogenesis and principles of treatment. Ann Surg 1974;179:697-711. 